\title{
Programmed death-ligand 1 expression and tumor-infiltrating lymphocytes in non-small cell lung cancer: association with clinicopathologic parameters
}

\author{
Gaurav Garg ${ }^{1}$, Kuruswamy Thurai Prasad ${ }^{2}$, Navneet Singh ${ }^{2}$, Parul Gupta $^{1}$, Valliappan Muthu², Ashim Das ${ }^{1}$, Amanjit Bal $^{1}$ \\ Departments of ${ }^{1}$ Histopathology and ${ }^{2}$ Pulmonary and Critical Care Medicine, Postgraduate Institute of Medical Education and Research, Chandigarh, India
}

\begin{abstract}
Background: Data on the prevalence of programmed death-ligand 1 (PD-L1) expression and tumor-infiltrating lymphocytes (TILs) in non-small cell lung cancer (NSCLC) and their clinical significance in Indian patients are limited. Methods: Newly diagnosed NSCLC cases (adenocarcinoma or squamous cell carcinoma [SqCC] histology) were included in the present study. The TILs were evaluated based on morphology on hematoxylin and eosin-stained slides. PD-L1 expression in tumors was assessed using immunohistochemistry with rabbit monoclonal antibody (SP263) on the Ventana automated immunostainer. Tumors with PD-L1 expression $>50 \%$ on tumor cells were considered PD-L1-positive. Tumors in which TILs occupy $>25 \%$ of stroma were considered to have high TILs. The association of PD-L1 expression and TILs with various clinical parameters including overall survival (OS) was investigated. Results: The present study included 128 cases of NSCLC (67 adenocarcinoma, $61 \mathrm{SqCC}$ ). PD-L1 positivity was observed in $17.2 \%$ of the patients with NSCLC. Baseline characteristics of PD-L1-positive subjects were similar to PD-L1-negative subjects except for a higher prevalence of liver metastasis ( $18.2 \%$ vs. $2.8 \% ; p=.018)$ and a higher probability of diagnosis from extrapulmonary biopsies. High TILs were observed in $26.6 \%$ of the subjects. However, PD-L1 expression and high TIL did not affect OS. Conclusions: PD-L1 positivity and high TILs were observed in $20 \%$ and $25 \%$ of the patients with NSCLC, respectively, however, neither were predictors of survival in SqCC.
\end{abstract}

Key Words: Lung neoplasms; Non-small cell lung carcinoma; PD-L1; Immunotherapy; Tumor-infiltrating lymphocytes

Received: November 10, 2020 Revised: July 13, 2021 Accepted: August 8, 2021

Corresponding Author: Amanjit Bal, MD, DNB, Department of Histopathology, Postgraduate Institute of Medical Education and Research, 5th Floor, Research Block-A, Chandigarh 160012, India

Tel: +91-172-2755104, Fax: +91-172-2744401, E-mail: docaman5@hotmail.com

Interaction of tumor cells with their microenvironment may result in infiltration of the tumor by immune cells of the host. Although such immune cell infiltration does not universally occur in all tumors, when present, these tumor-infiltrating lymphocytes (TILs) may provide tumor-specific immune response to prevent further tumorigenesis. However, tumor cells may evade host immunity through the expression of several transmembrane proteins including programmed death-ligand 1 (PD-L1). PD-L1-targeted therapy is a form of immunotherapy which targets the components of the PD-L1 pathway to prevent downregulation of anti-tumoral immunity. Unfortunately, PD-L1-targeted therapy is effective in $<50 \%$ of non-small cell lung cancers (NSCLCs) despite the presence of PD-L1 expression. However, in general, better results are observed in patients with higher PD-L1 expression [1-4]. Therefore, testing for PD-L1 expression using immunohistochemistry is often performed before initiating PD-L1-targeted therapy. The prevalence of PD-L1 positivity in NSCLC varies widely across studies performed in different parts of the world [2-5]. A large part of this difference could be attributed to methodological differences which is supported by the results of a recent multinational study (EXPRESS) which showed similar PD-L1 expression in advanced NSCLCs across various geographical regions when a uniform testing strategy was used [6]. However, the reported prevalence of PD-L1 expression in Indian patients with NSCLC, albeit with different methods, is much lower $[7,8]$. Furthermore, the PD-L1 expression in NSCLC has been associated with certain clinicopathological characteristics and poor clinical outcomes in some but not all studies [5]. The intensity of tumor infiltration by TILs has been associated with survival $[9,10]$. In the present study, the prevalence and prog- 
nostic significance of PD-L1 expression in a cohort of NSCLC patients from North India were reported.

\section{MATERIALS AND METHODS}

\section{Patients}

This was a single institute, prospective, observational study conducted over 2 years. Consecutive patients with newly diagnosed NSCLC with adenocarcinoma or squamous cell carcinoma (SqCC) histology were considered for inclusion in this study. Subjects with a biopsy sample insufficient for the necessary histopathological analyses were excluded from the study.

\section{Histopathological examination}

All specimens were processed for paraffin sections for routine hematoxylin and eosin $(\mathrm{H} \& \mathrm{E})$ staining and immunohistochemistry. The specimens included lung biopsies (bronchoscopic biopsies and computed tomography [CT]/positron emission tomography (PET)-guided biopsies) and biopsies from metastatic sites (lymph nodes, pleura, bone, and brain). Cytology samples were not included in the study. Based on $\mathrm{H} \& \mathrm{E}$, the tumors were categorized as adenocarcinoma or SqCC as defined by the 2015 World Health Organization classification of lung tumors [11]. Tumors in which histological subtyping on the basis of routine $\mathrm{H} \& \mathrm{E}$ staining was difficult, immunohistochemistry with p63, thyroid transcription factor 1 , cytokeratin (CK) $5 / 6, \mathrm{CK} 7$, neuron-specific enolase, and $\mathrm{CD} 56$ was used.

\section{Tumor-infiltrating lymphocytes}

The TILs were evaluated based on morphology on H\&Estained slides. Only lymphocytes and plasma cells were included in the scoring. Necrotic areas within the tumor and alveolar macrophages were not included in the scoring [12]. TILs were assessed in five areas of the tumor to determine the mean TIL score. The TIL score was assigned based on the proportion of tumor stroma occupied by TILs (TIL0, 0 to $\leq 5 \%$; TIL1, > $5 \%$ to $\leq 25 \%$; TIL2, $>25$ to $\leq 50 \%$; TIL3, > 50\%). Subjects with a score of TIL0 were considered to have low TILs and subjects with a score of TIL2 or TIL3 were considered to have high TILs.

\section{PD-L1 expression}

PD-L1 expression in tumors was assessed using immunohistochemistry with rabbit monoclonal antibody (SP263) on the Ventana automated immunostainer. Detection was optimized with the OptiView DAB IHC Detection Kit (Ventana Medical Systems, Tucson, AZ, USA). For positive controls, sections of the human placenta, as recommended in the data sheet of SP263 antibody (Fig. 1A) as well as human tonsil, were included in each batch. A negative control was run for each case. At least 100 viable tumor cells were scored. Tumor cells were considered to express PD-L1 when they showed complete or partial membranous staining with or without cytoplasmic staining of any intensity. PD-L1 expression on tumor cells was assigned scores based on the proportion of tumor cells (TC0, 0 to $<1 \%$; TC1, $\geq 1$ to $\leq 50 \%$; TC2, > 50\%) expressing PD-L1. Tumors with a PD-L1 expression on tumor cells $>50 \%$ (TC2) were considered PDL1-positive.

\section{Mutation analysis}

Epidermal growth factor receptor (EGFR) mutations and anaplastic lymphoma kinase (ALK) rearrangements were evaluated in subjects with adenocarcinoma histology. EGFR mutation analysis was performed using real-time PCR (EnteroGen, Agility Biotech, Los Angeles, CA, USA). ALK rearrangements were identified on immunohistochemistry performed on a Ventana BenchMark XT automated slide-processing system using the D5F3 clone [13].

\section{Clinical details}

The following clinical parameters were recorded at baseline: age, sex, smoking status, body mass index, performance status, and TNM. Performance status was evaluated using the Eastern Cooperative Oncology Group scale [14]. Contrast-enhanced CT scan of the thorax and upper abdomen (including the liver and adrenals) or whole-body PET was obtained for baseline staging evaluation in all patients. Tumor staging was performed using the seventh edition of the American Joint Committee on Cancer (AJCC) TNM classification [15]. Tumor staging was also performed using the eighth edition of the AJCC TNM classification in 106 patients (Supplementary Table S1). Because the proportion of patients between the seventh and eighth edition of the AJCC TNM classification was not significantly different, and to ensure completeness of data, the staging in the seventh edition of the AJCC TNM classification was used in the present study.

Subjects were treated with chemotherapy, targeted therapy, immunotherapy, radiotherapy, or surgery as indicated by tumor histopathology, mutation status, and clinical status, as previously described [16]. Briefly, subjects with adenocarcinoma without any driver mutation were treated with pemetrexedbased platinum doublet followed by maintenance pemetrexed therapy until disease progression [17]. Subjects with squamous histology were treated with docetaxel or gemcitabine-based 
platinum doublet. All patients receiving chemotherapy were administered at least four cycles of chemotherapy before response assessment using the Response Evaluation Criteria in Solid Tumors ver. 1.0 [18]. Subjects with sensitizing EGFR mutation or $A L K$ rearrangement were treated with appropriate EGFR tyrosine kinase or ALK inhibitors, respectively [19,20].

\section{Statistical analysis}

Continuous variables were expressed as the mean and standard deviation and categorical values were expressed as the numbers and percentages. The differences between continuous and categorical variables were analyzed using the Mann-Whitney $\mathrm{U}$ test and the chi-square test (or Fisher exact test), respectively. Overall survival (OS) was defined as the time between initial diagnosis and date of death or last follow-up. The cutoff date for survival analysis was November 30, 2018. Survival curves were generated using the Kaplan-Meir method and were compared using the $\log$-rank test. Multivariate analysis to identify predictors of survival was performed using the Cox proportional hazard model. Statistical analyses were performed using the commercial statistical package SPSS (IBM SPSS Statistics, ver. 22, IBM Corp., Armonk, NY, USA). A p-value $<0.05$ was considered statistically significant.

\section{RESULTS}

A total of 128 cases of NSCLC were included in the present study (Table 1); 103 (80.5\%) were males and the median age of the study population was $61 \pm 15$ years. The majority of patients had advanced disease (84.1\% were stage IIIB/IV) and $44.8 \%$ of the patients had extrathoracic disease at diagnosis. Tissue samples (small biopsies: endobronchial biopsies, transbronchial biopsies, and imaging-guided core biopsies) were predominantly obtained from the lung $(82.4 \%)$ and metastatic lymph nodes (8\%), and $9.6 \%$ were resection samples. Adenocarcinoma and SqCC were observed in $52.3 \%$ and $47.7 \%$ of the subjects, respectively. A larger proportion of patients who were younger, female, non-smokers, and presented with advanced disease had adenocarcinoma. EGFR and $A L K$ alterations were observed in $6.2 \%$ and $5.4 \%$ of the subjects, respectively. Chemotherapy $(74.2 \%)$ was the most commonly used first-line therapy. Due to economic constraints, PD-L1-targeted therapy despite PD-L1 expression could not be provided to any patient in this study.

Based on immunohistochemistry, the majority of patients had PD-L1 expression on $<1 \%$ of tumor cells (TC0, 61.7\%) (Table 2, Fig. 1). PD-L1 expression on $\geq 1 \%$ of tumor cells (TC1
Table 1. Baseline clinical characteristics

\begin{tabular}{|c|c|c|c|c|}
\hline Characteristic & $\begin{array}{c}\text { Total } \\
(n=128)\end{array}$ & $\begin{array}{c}\text { Adenocarcinoma } \\
(n=67)\end{array}$ & $\begin{array}{l}\text { SqCC } \\
(n=61)\end{array}$ & $p$-value \\
\hline Age (yr) & $59.5 \pm 11.1$ & $57.5 \pm 11.4$ & $61.8 \pm 10.5$ & .047 \\
\hline Male sex & $103(80.5)$ & $45(67.2)$ & $58(95.1)$ & $<.001$ \\
\hline Smokers ${ }^{a}$ & $95(74.2)$ & 39 (58.2) & $56(94.9)$ & $<.001$ \\
\hline $\begin{array}{l}\text { Body mass index } \\
\left(\mathrm{kg} / \mathrm{m}^{2}\right)\end{array}$ & $20.3 \pm 4.0$ & $20.9 \pm 3.9$ & $19.7(4.0)$ & .093 \\
\hline ECOG PS score $\geq 2$ & $19(14.8)$ & $9(13.4)$ & $10(16.4)$ & .638 \\
\hline $\begin{array}{l}\text { TNM stage } \\
\text { at diagnosis }^{\mathrm{a}}\end{array}$ & & & & $.024^{b}$ \\
\hline । & $2(1.6)$ & $2(3.0)$ & 0 & \\
\hline$\|$ & $5(4.0)$ & $2(3.0)$ & $3(5.1)$ & \\
\hline$\| I A$ & $13(10.3)$ & $2(3.0)$ & $11(18.6)$ & \\
\hline$\| \mathrm{IIB}$ & $27(21.4)$ & $9(13.4)$ & $18(30.5)$ & \\
\hline IV & $79(62.7)$ & $52(77.6)$ & $27(45.8)$ & \\
\hline $\begin{array}{l}\text { Extrathoracic } \\
\text { disease }^{c}\end{array}$ & $56(44.8)$ & $41(61.2)$ & $15(25.9)$ & $<.001$ \\
\hline Biopsy site $^{c}$ & & & & $.087^{\mathrm{d}}$ \\
\hline Lung & $103(82.4)$ & $50(75.8)$ & $53(89.8)$ & \\
\hline Lymph node & $10(8.0)$ & $6(9.1)$ & $4(6.8)$ & \\
\hline Pleura & $5(4.0)$ & $5(7.6)$ & 0 & \\
\hline Other & $7(5.6)$ & $5(7.6)$ & $2(3.4)$ & \\
\hline EGFR-positive & $8(6.2)$ & $8(11.9)$ & 0 & .005 \\
\hline ALK-positive & $7(5.4)$ & $7(10.4)$ & 0 & .009 \\
\hline First-line treatment & & & & .011 \\
\hline Chemotherapy & $95(74.2)$ & 49 (73.1) & $46(75.4)$ & \\
\hline Targeted therapye & $16(12.5)$ & $13(19.4)$ & $3(4.9)$ & \\
\hline Other & $17(13.3)$ & $5(7.5)$ & $12(19.7)$ & \\
\hline
\end{tabular}

Values are presented as mean \pm SD or number (\%).

SqCC, squamous cell carcinoma; ECOG PS, Eastern Cooperative Oncology Group performance status; EGFR, epidermal growth factor receptor; ALK, anaplastic lymphoma kinase; SD, standard deviation.

aData were not available in two patients; ${ }^{\text {b}}$ For comparison of stage IIIB or IV between adenocarcinomas and SqCCs; 'Data were not available in three patients; dFor comparison of lung biopsy between adenocarcinomas and SqCCs; ePatients who had a high probability of underlying EGFR mutation (e.g., non-smoking females) in whom molecular testing could not be performed (inadequate tissue for molecular analysis in the initial sample with the patient unwilling or unfit for a repeat invasive procedure) and whose performance status did not permit the use of chemotherapy were provided targeted therapy on compassionate grounds. Therefore, the number of patients with driver mutations and the number of patients who received targeted therapy was not equal.

or TC2) was observed in $38.3 \%$ of the subjects. However, this did not differ between subjects with adenocarcinoma or SqCC $(\mathrm{p}=.335)$. TIL1 $(31.3 \%)$ and TIL0 $(23.4 \%)$ were the most commonly observed TIL scores (Table 2, Fig. 2). High TILs (TIL occupying $>25 \%$ of tumor stroma, TIL2 or TIL3) were observed in $26.6 \%$ of patients. Infiltration $>50 \%$ of tumor stroma by TILs (TIL3) occurred in the least number of cases (7.8\%). Difference in the TIL scores between adenocarcinoma and SqCC was not observed $(\mathrm{p}=.126)$. The baseline characteristics were not different between subjects with high and low TILs (data not shown). A higher proportion of patients with PD-L1 expression 
Table 2. Histopathological characteristics of the overall population

\begin{tabular}{lcrrr}
\hline Variable & $\begin{array}{c}\text { Total } \\
(n=128)\end{array}$ & $\begin{array}{c}\text { Adenocarcinoma } \\
(n=67)\end{array}$ & $\begin{array}{c}\text { SqCC } \\
(n=61)\end{array}$ & p-value \\
\hline PD-L1 TC score & & & & $.335^{\mathrm{a}}$ \\
TC0 & $79(61.7)$ & $44(65.7)$ & $35(57.4)$ & \\
TC1 & $27(21.1)$ & $8(11.9)$ & $19(31.1)$ & \\
TC2 & $22(17.2)$ & $15(22.4)$ & $7(11.5)$ & \\
TIL score $^{b}$ & & & & $.126^{c}$ \\
TIL0 & $30(23.4)$ & $18(26.9)$ & $12(19.7)$ & \\
TIL1 & $40(31.3)$ & $22(32.8)$ & $18(29.5)$ & \\
TIL2 & $24(18.8)$ & $10(14.9)$ & $14(23.0)$ & \\
TIL3 & $10(7.8)$ & $4(6.0)$ & $6(9.8)$ & \\
\hline
\end{tabular}

Values are presented as number (\%).

The TC score was assigned based on the proportion of tumor cells expressing PD-L1 (TCO: $<1 \%$, TC1: $\geq 1$ but $\leq 50 \%$, TC2: $>50 \%$ ). The TIL score was assigned based on the proportion of tumor stroma occupied by TILS (TIL0: $\leq 5 \%$, TIL1: $\leq 25 \%$, TIL2: > 25 but $\leq 50 \%$, TIL3: > 50\%).

SqCC, squamous cell carcinoma; PD-L1, programmed death-ligand 1; TC, tumor cell; TILs, tumor-infiltrating lymphocytes.

aFor comparison of the proportion of subjects with PD-L1 expression on $\geq 1 \%$ of tumor cells (TC1 or TC2) between adenocarcinomas and SqCCs; ' ${ }^{\mathrm{TII}}$ score was not available for 26 patients; 'For comparison of the proportion of subjects with $\geq 25 \%$ of tumor stroma infiltrated by TILs (TIL2 or TIL3) between adenocarcinomas and SqCCs.
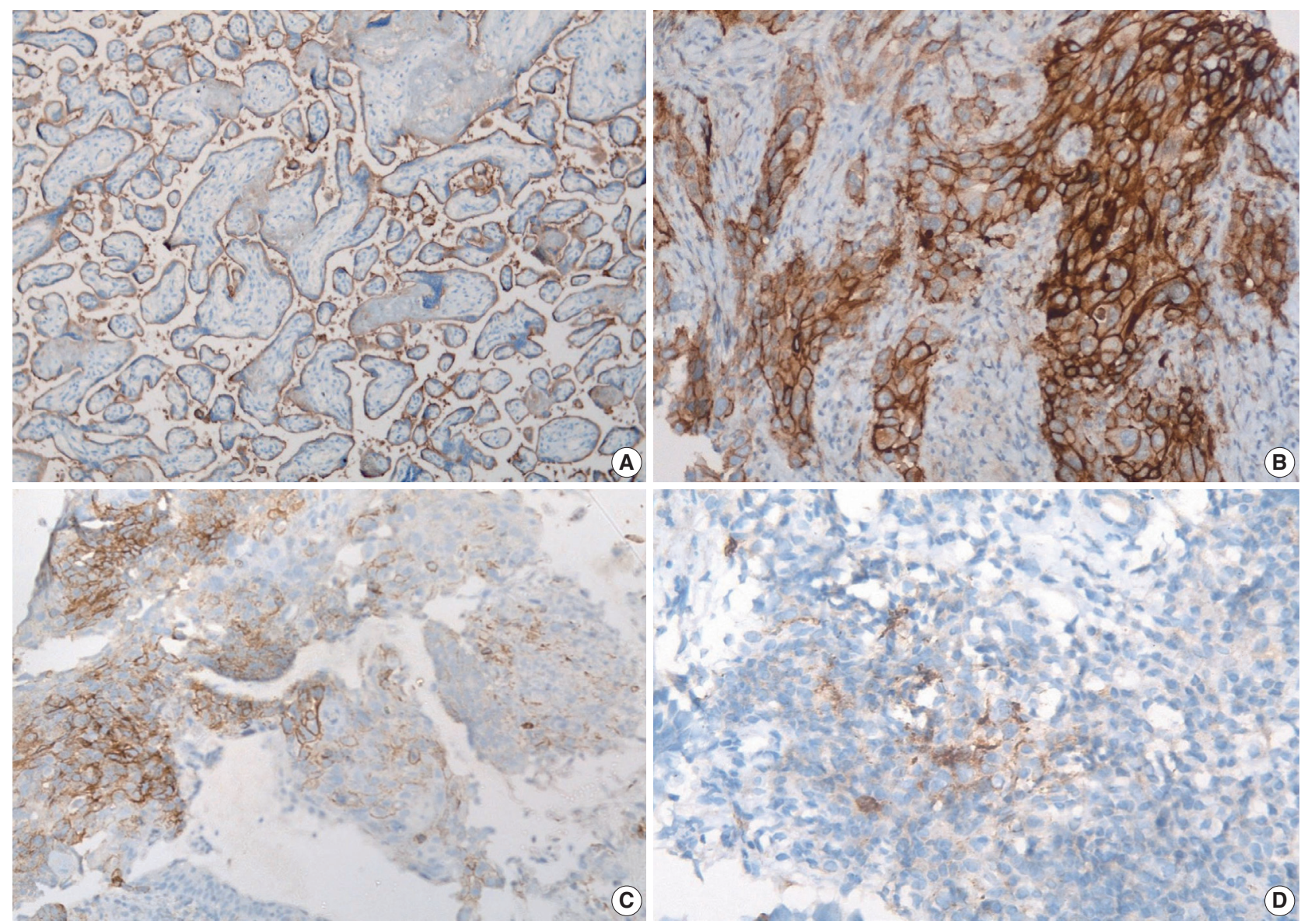

Fig. 1. Photomicrographs (programmed death-ligand 1 [PD-L1] staining using SP263 clone) showing positive control (PD-L1 staining in placenta) (A), TC2 (PD-L1 expression on > 50\% of tumor cells [TCs]) (B), TC1 (PD-L1 expression on 1\%-50\% TCs) (C), and TC0 (PD-L1 expression on $<1 \%$ of TCS) (D). 

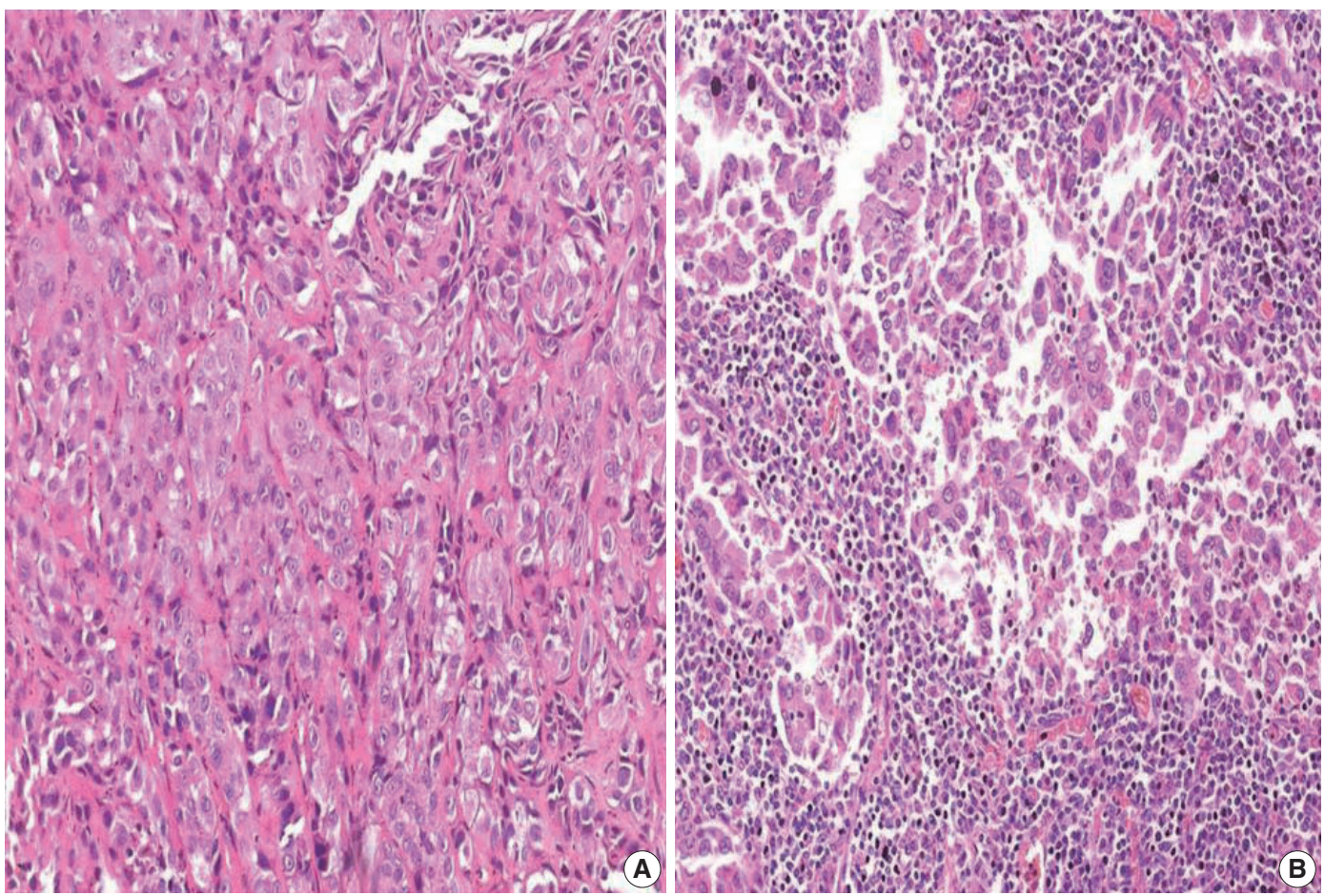

Fig. 2. Photomicrographs $(\times 200)$ of hematoxylin and eosin staining showing TILO $(0 \%-5 \%$ of tumor stroma occupied by tumor-infiltrating lymphocytes [TILs]) (A), TIL3 (> 50\% of tumor stroma occupied by TILs) (B).

\section{DISCUSSION}

In this study, $17.2 \%$ of the patients with NSCLC could be labelled as PD-L1-positive (PD-L1 expression on tumor cells $>50 \%$ ). PD-L1 expression $\geq 1 \%$ on tumor cells was observed in $38.3 \%$ of the subjects. PD-L1-positive subjects had a higher prevalence of liver metastasis and were more often diagnosed using biopsy samples obtained from extrapulmonary sites. The PD-L1 expression did not affect survival in the study population. High TILs (TILs occupying $>25 \%$ of tumor stroma) were observed in $26.6 \%$ of the subjects, however, it did not affect survival. A larger proportion of patients with higher PD-L1 expression on tumor cells was more associated with subjects with high TILs than low TILs.

The assessment of PD-L1 expression based on immunohistochemistry is complicated by several factors including intra-tumor heterogeneity and inter-assay variation [21]. Therefore, the estimated prevalence of PD-L1-positive NSCLCs varies widely (7\%75\%) $[5,22,23]$. In the multinational EXPRESS study, PD-L1 expression on $\geq 50 \%$ and $\geq 1 \%$ of tumor cells was observed in $22 \%$ and $52 \%$ of patients with advanced NSCLC, respectively, using the $22 \mathrm{C} 3$ pharmDx kit [6]. In the present study, PD-L1 positivity was observed in $17.2 \%$ of NSCLCs (38.3\% had PDL1 expression $\geq 1 \%$ on tumor cells). The SP263 antibody clone was used because its performance is comparable or better than the other available antibody clones [24-26]. In prior studies from India, a PD-L1 positivity rate of 27\% (> 5\% PD-L1 expression on tumor cells) and $34 \%$ ( $\geq 1 \%$ PD-L1 expression on tumor cells) was reported using the SP142 and SP263 clones, respectively $[7,8]$. Overall, the reported rates of PD-L1 expression in lung cancer in India appears to be less than international estimates. Whether this difference is due to methodological differences or true geographic differences is unclear.

PD-L1 expression has been associated with male sex, smoking, advanced tumor stage, SqCC histology, and EGFR mutation $[5,22,27,28]$. In the present study, association between PD-L1 expression and sex, smoking, or TNM stage, was not found. Liver metastasis was more common in subjects who were PD-L1-positive. The PD-L1 positivity rate was not affected by histology.

The association between PD-L1 expression and survival is controversial $[5,7,22]$. In a meta-analysis of 41 studies, PD-L1 expression was associated with poor survival in NSCLCs, specifically in subgroups of patients with adenocarcinoma or early disease [5]. In contrast, another study showed that PD-L1 expression resulted in worse prognosis in $\mathrm{SqCC}$ but not adenocarcinoma [22]. Furthermore, in another study, PD-L1 positivity in immune cells was found associated with better prognosis in resected NSCLCs [7]. In the present study, PD-L1 expression was not as- 
Table 3. Difference in clinical characteristics between PD-L1-positive and PD-L1-negative subjects

\begin{tabular}{|c|c|c|c|}
\hline Variable & $\begin{array}{l}\text { PD-L1 negative } \\
\quad(n=106)\end{array}$ & $\begin{array}{l}\text { PD-L1 positive } \\
(n=22)\end{array}$ & $p$-value \\
\hline Age (yr) & $59.6 \pm 10.6$ & $59.5 \pm 13.6$ & .934 \\
\hline Male sex & $88(83.0)$ & $15(68.2)$ & .240 \\
\hline Smokers $^{a}$ & $82(77.3)$ & $13(59.1)$ & .051 \\
\hline Body mass index $\left(\mathrm{kg} / \mathrm{m}^{2}\right)$ & $20.5 \pm 4.0$ & $19.3 \pm 3.7$ & .308 \\
\hline ECOG score $\geq 2$ & $17(16.0)$ & $2(9.1)$ & .525 \\
\hline TNM stage at diagnosis ${ }^{a}$ & & & .649 \\
\hline । & $2(1.9)$ & 0 & \\
\hline$\|$ & $4(3.8)$ & $1(4.5)$ & \\
\hline$\| \mathrm{A}$ & $11(10.4)$ & $2(9.1)$ & \\
\hline$\| I I B$ & $25(23.6)$ & $2(9.1)$ & \\
\hline IV & $62(58.5)$ & $17(77.3)$ & \\
\hline $\begin{array}{l}\text { Extrathoracic disease } \\
\text { at baseline }{ }^{b}\end{array}$ & $44(41.5)$ & $12(54.5)$ & .311 \\
\hline Liver metastasis & $3(2.8)$ & $4(18.2)$ & .018 \\
\hline Biopsy site ${ }^{b}$ & & & .022 \\
\hline Lung & $91(85.8)$ & $12(54.5)$ & \\
\hline Lymph node & $6(5.7)$ & $4(18.2)$ & \\
\hline Pleura & $3(2.8)$ & $2(9.1)$ & \\
\hline Other & $4(3.8)$ & $3(13.6)$ & \\
\hline Histology & & & .102 \\
\hline Adenocarcinoma & $52(49.1)$ & $15(68.2)$ & \\
\hline Squamous cell carcinoma & $54(50.9)$ & $7(31.8)$ & \\
\hline EGFR-positive & $5(4.7)$ & $3(13.6)$ & .138 \\
\hline ALK-positive & $5(4.7)$ & $2(9.1)$ & .345 \\
\hline First-line treatment & & & .664 \\
\hline Chemotherapy & $80(75.5)$ & $15(68.2)$ & \\
\hline Targeted therapy & $12(11.3)$ & $4(18.2)$ & \\
\hline None & 14 (13.2) & $3(13.6)$ & \\
\hline TIL score $(\%)^{c}$ & & & .890 \\
\hline $0-5$ & $23(28.0)$ & $7(31.8)$ & \\
\hline $6-25$ & $33(40.2)$ & $7(31.8)$ & \\
\hline $26-50$ & $18(22.0)$ & $6(27.3)$ & \\
\hline$>50$ & $8(9.8)$ & $2(9.1)$ & \\
\hline
\end{tabular}

Values are presented as mean \pm SD or number (\%).

Tumors with PD-L1 expression $>50 \%$ in tumor cells (TC2) were considered PD-L1-positive.

PD-L1, programmed death-ligand 1; ECOG, Eastern Cooperative Oncology Group; EGFR, epidermal growth factor receptor; ALK, anaplastic lymphoma kinase; TIL, tumor-infiltrating lymphocyte; SD, standard deviation.

aData were not available in two patients; 'Data were not available in three patients; 'TIL score was not available for 24 patients.

sociated with survival.

Infiltration $\geq 20 \%$ of tumor stroma with TILs has been observed in 50\% of subjects with NSCLCs [26]. Higher TILs have been associated with higher PD-L1 expression and better survival in NSCLCs $[9,10,29]$. In the present study, approximately onefourth of the tumors showed high TILs (infiltration $>25 \%$ of tumor stroma by lymphocytes TIL2 or TIL3). Although high TILs were associated with higher PD-L1 expression in both TILs and tumor cells, high TILs did not affect survival in our study cohort.

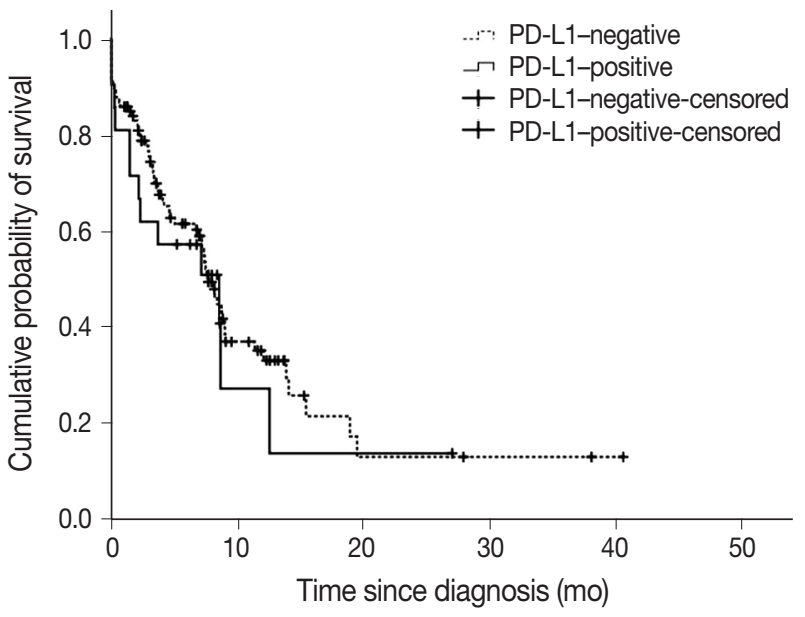

Fig. 3. Kaplan-Meir plots for overall survival (OS). OS of programmed death-ligand 1 (PD-L1)-positive subjects was not different from PD-L1-negative subjects. The median $(95 \%$ confidence interval) OS was 7.6 months (6.5-8.8) in subjects who were PDL1-negative compared with 8.5 months $(1.1-16.0)$ in subjects who were PD-L1-positive (log-rank $\mathrm{P}=.584)$.

Table 4. Multivariate analysis for predictors of OS

\begin{tabular}{lcc}
\hline Variable & $\mathrm{HR}(95 \% \mathrm{Cl})$ & $\mathrm{p}$-value \\
\hline Age & $1.00(0.98-1.03)$ & .708 \\
Female sex & $0.39(0.16-0.98)$ & .046 \\
Smoking & $0.84(0.36-2.00)$ & .682 \\
ECOG PS $\geq 2$ & $0.66(0.30-1.45)$ & .297 \\
TNM stage IIIB or IV & $1.68(0.82-3.44)$ & .154 \\
Adenocarcinoma histology & $0.78(0.46-1.33)$ & .358 \\
PD-L1-positive & $1.47(0.78-2.77)$ & .231 \\
\hline
\end{tabular}

Tumors with PD-L1 expression > 50\% on tumor cells (TC2) or PD-L1 expression of $1 \%-50 \%$ on tumor cells (TC1) with PD-L1 expression $>10 \%$ in TILS (IC2) were considered PD-L1-positive.

OS, overall survival; HR, hazard ratio; $\mathrm{Cl}$, confidence interval; ECOG PS, Eastern Cooperative Oncology Group performance status; PD-L1, programmed death-ligand 1; TIL, tumor-infiltrating lymphocytes.

This was a single-center study with a relatively small sample size, thus, the results, especially subgroup analyses, should be interpreted cautiously. The TNM stage was not a prognostic factor in the present study for several reasons. The study had a relatively small sample size $(\mathrm{n}=128)$. A disproportionately larger proportion of patients had stage IV disease $(62.7 \%$ with stage IV disease in this study compared with $51.1 \%$ with stage IV disease in a previous analysis from our center with a much larger sample size $(n=1,501)$ [14]. The EGFR and ALK positivity rate observed in this study was considerably lower than what is generally observed at our center possibly due to selection bias [30]. Subjects who had undergone mutation testing could have been excluded from this study because they might have been left with inadequate tissue specimen for additional histopathological analyses. 
Furthermore, a considerable proportion of lung cancer patients diagnosed based on cytology samples were not included in this study.

In conclusion, the present study results showed PD-L1 positivity and high TILs can be observed in approximately one-fifth and one-fourth of the patients with NSCLC, respectively. However, PD-L1 expression or high TILs did not affect the OS in our study cohort.

\section{Supplementary Information}

The Data Supplement is available with this article at https://doi.org/10.4132/ jptm.2021.08.08.

\section{Ethics Statement}

Written informed consent was obtained from all the study participants. The study was approved by the Institutional Ethics Review Committee (Reference number: NK/4398/MD/381).

\section{Availability of Data and Material}

The datasets generated or analyzed during the study are available from the corresponding author on reasonable request.

\section{Code Availability}

Not applicable.

\section{ORCID}

Gaurav Garg Kuruswamy Thurai Prasad Navneet Singh Parul Gupta Valliappan Muthu Ashim Das Amanjit Bal https://orcid.org/0000-0002-1658-9441 https://orcid.org/0000-0001-7690-6595 https://orcid.org/0000-0002-8389-0701 https://orcid.org/0000-0001-7035-1752 https://orcid.org/0000-0003-0410-8468 https://orcid.org/0000-0002-2031-7000 https://orcid.org/0000-0002-1457-8589

\section{Author Contributions}

Conceptualization of study: AB. Data curation: GG, KTP, NS, PG. Formal analysis: $\mathrm{AB}, \mathrm{AD}, \mathrm{VM}$. Investigation: GG. Methodology: $\mathrm{AB}, \mathrm{AD}, \mathrm{VM}, \mathrm{GG}$, PG. Resources: AB, NS, KTP, GG, PG. Supervision: AB. Validation: AB, $A D$, KTP, NS. Writing—original draft: AB, GG. Writing—review \& editing: $\mathrm{AB}, \mathrm{KTP}, \mathrm{PG}, \mathrm{AD}$, NS. Approval of final manuscript: all authors.

\section{Conflicts of Interest}

The authors declare that they have no potential conflicts of interest.

\section{Funding Statement}

No funding to declare.

\section{References}

1. Mok TSK, Wu YL, Kudaba I, et al. Pembrolizumab versus chemotherapy for previously untreated, PD-L1-expressing, locally advanced or metastatic non-small-cell lung cancer (KEYNOTE-042): a randomised, open-label, controlled, phase 3 trial. Lancet 2019; 393: 1819-30.

2. Socinski MA, Jotte RM, Cappuzzo F, et al. Atezolizumab for first-line treatment of metastatic nonsquamous NSCLC. N Engl J Med 2018;
378: 2288-301.

3. Gandhi L, Rodriguez-Abreu D, Gadgeel S, et al. Pembrolizumab plus chemotherapy in metastatic non-small-cell lung cancer. N Engl J Med 2018; 378: 2078-92.

4. Paz-Ares L, Luft A, Vicente D, et al. Pembrolizumab plus chemotherapy for squamous non-small-cell lung cancer. N Engl J Med 2018; 379: 2040-51.

5. Zhang M, Li G, Wang Y, et al. PD-L1 expression in lung cancer and its correlation with driver mutations: a meta-analysis. Sci Rep 2017; 7: 10255.

6. Dietel M, Savelov N, Salanova R, et al. Real-world prevalence of programmed death ligand 1 expression in locally advanced or metastatic non-small-cell lung cancer: the global, multicenter EXPRESS study. Lung Cancer 2019; 134: 174-9.

7. Vallonthaiel AG, Malik PS, Singh V, et al. Clinicopathologic correlation of programmed death ligand-1 expression in non-small cell lung carcinomas: a report from India. Ann Diagn Pathol 2017; 31: 56-61.

8. Kumar M, Guleria B, Swamy S, Soni S. Correlation of programmed death-ligand 1 expression with gene expression and clinicopathological parameters in Indian patients with non-small cell lung cancer. Lung India 2020; 37: 145-50.

9. Brambilla E, Le Teuff G, Marguet S, et al. Prognostic effect of tumor lymphocytic infiltration in resectable non-small-cell lung cancer. J Clin Oncol 2016; 34: 1223-30.

10. Bremnes RM, Busund LT, Kilvaer TL, et al. The role of tumor-infiltrating lymphocytes in development, progression, and prognosis of non-small cell lung cancer. J Thorac Oncol 2016; 11: 789-800.

11. Travis WD, Brambilla E, Nicholson AG, et al. The 2015 World Health Organization classification of lung tumors: impact of genetic, clinical and radiologic advances since the 2004 classification. J Thorac Oncol 2015; 10: 1243-60.

12. Hendry S, Salgado R, Gevaert T, et al. Assessing tumor-infiltrating lymphocytes in solid tumors: a practical review for pathologists and proposal for a standardized method from the International Immuno-Oncology Biomarkers Working Group: Part 2: TILs in melano$\mathrm{ma}$, gastrointestinal tract carcinomas, non-small cell lung carcinoma and mesothelioma, endometrial and ovarian carcinomas, squamous cell carcinoma of the head and neck, genitourinary carcinomas, and primary brain tumors. Adv Anat Pathol 2017; 24: 311-35.

13. Bal A, Singh N, Agarwal P, Das A, Behera D. ALK gene rearranged lung adenocarcinomas: molecular genetics and morphology in cohort of patients from North India. APMIS 2016; 124: 832-8.

14. Prasad KT, Kaur H, Muthu V, Aggarwal AN, Behera D, Singh N. Interconversion of two commonly used performance tools: an analysis of 5844 paired assessments in 1501 lung cancer patients. World J Clin Oncol 2018; 9: 140-7.

15. Goldstraw P, Crowley J, Chansky K, et al. The IASLC Lung Cancer Staging Project: proposals for the revision of the TNM stage groupings in the forthcoming (seventh) edition of the TNM classification of malignant tumours. J Thorac Oncol 2007; 2: 706-14.

16. Singh N, Aggarwal AN, Behera D. Management of advanced lung cancer in resource-constrained settings: a perspective from India. Expert Rev Anticancer Ther 2012; 12: 1479-95.

17. Prasad KT, Muthu V, Biswas B, et al. Utility and safety of maintenance chemotherapy in advanced non-small cell lung cancer across various performance status categories: real-world experience. Curr Probl Cancer 2020; 44: 100565. 
18. Therasse P, Arbuck SG, Eisenhauer EA, et al. New guidelines to evaluate the response to treatment in solid tumors. European Organization for Research and Treatment of Cancer, National Cancer Institute of the United States, National Cancer Institute of Canada. J Natl Cancer Inst 2000; 92: 205-16.

19. Garg A, Batra U, Choudhary P, et al. Clinical predictors of response to EGFR-tyrosine kinase inhibitors in EGFR-mutated non-small cell lung cancer: a real-world multicentric cohort analysis from India. Curr Probl Cancer 2020; 44: 100570.

20. Patel A, Batra U, Prasad KT, et al. Real world experience of treatment and outcome in $A L K$-rearranged metastatic nonsmall cell lung cancer: a multicenter study from India. Curr Probl Cancer 2020; 44: 100571.

21. McLaughlin J, Han G, Schalper KA, et al. Quantitative assessment of the heterogeneity of PD-L1 expression in non-small-cell lung cancer. JAMA Oncol 2016; 2: 46-54.

22. Sorensen SF, Zhou W, Dolled-Filhart M, et al. PD-L1 expression and survival among patients with advanced non-small cell lung cancer treated with chemotherapy. Transl Oncol 2016; 9: 64-9.

23. Calles A, Liao X, Sholl LM, et al. Expression of PD-1 and its ligands, PD-L1 and PD-L2, in smokers and never smokers with KRAS-mutant lung cancer. J Thorac Oncol 2015; 10: 1726-35.

24. Hirsch FR, McElhinny A, Stanforth D, et al. PD-L1 immunohistochemistry assays for lung cancer: results from phase 1 of the Blue- print PD-L1 IHC Assay Comparison Project. J Thorac Oncol 2017; 12: 208-22.

25. Parra ER, Villalobos P, Mino B, Rodriguez-Canales J. Comparison of different antibody clones for immunohistochemistry detection of programmed cell death ligand 1 (PD-L1) on non-small cell lung carcinoma. Appl Immunohistochem Mol Morphol 2018; 26: 83-93.

26. Kim H, Kwon HJ, Park SY, Park E, Chung JH. PD-L1 immunohistochemical assays for assessment of therapeutic strategies involving immune checkpoint inhibitors in non-small cell lung cancer: a comparative study. Oncotarget 2017; 8: 98524-32.

27. Mandarano M, Bellezza G, Belladonna ML, et al. Assessment of TILs, IDO-1, and PD-L1 in resected non-small cell lung cancer: an immunohistochemical study with clinicopathological and prognostic implications. Virchows Arch 2019; 474: 159-68.

28. He Y, Rozeboom L, Rivard CJ, et al. PD-1, PD-L1 protein expression in non-small cell lung cancer and their relationship with tumor-infiltrating lymphocytes. Med Sci Monit 2017; 23: 1208-16.

29. Geng Y, Shao Y, He W, et al. Prognostic role of tumor-infiltrating lymphocytes in lung cancer: a meta-analysis. Cell Physiol Biochem 2015; 37: 1560-71.

30. Muthu V, Bal A, Gupta N, Prasad K, Behera D, Singh N. P2.03-053 A five-year audit of EGFR and ALK testing at a tertiary care centre in North India: more sensitive methods do make a difference! J Thorac Oncol 2017; 12: S2395. 